\title{
Mixed Finite Element Methods for Diffusion Equations on Nonmatching Grids
}

\author{
Yuri Kuznetsov \\ Department of Mathematics, University of Houston (kuz@math.uh.edu)
}

\begin{abstract}
Summary. The hybridization technique is applied to replace the macro-hybrid mixed finite element problem for the diffusion equation by the equivalent cell-based formulation. The underlying algebraic system is condensed by eliminating the degrees of freedom which represent the interface flux and cell pressure variables to the system containing the Lagrange multipliers variables. An approach to the numerical solution of the condensed system is briefly discussed.
\end{abstract}

\section{Introduction}

In this paper, we consider macro-hybrid mixed finite element method for the diffusion equation on nonmatching grids. The paper is organized as follows. The four-field macro-hybrid mixed formulation for the diffusion equation is given in Sect. 2.

In Sect. 3, we apply the hybridization technique to replace the macrohybrid formulation by the cell-based formulation and describe the condensation procedure to reduce the underlying algebraic system to the system for the Lagrange multipliers only. In Sect. 4, we briefly discuss an algebraic solution method for the condensed system.

\section{Problem formulation}

We consider the diffusion problem in the form of a system of the first order differential equations

$$
\begin{aligned}
K^{-1} \bar{u}+\operatorname{grad} p & =0 \\
\operatorname{div} \bar{u}+\quad c p & =f
\end{aligned}
$$

in a bounded connected polygonal (polyhedral) domain $\Omega$ in $\mathbb{R}^{d}, d=2(d=3)$ with homogeneous boundary conditions 


$$
\begin{array}{rll}
p=0 & \text { on } & \Gamma_{D}, \\
\bar{u} \cdot \bar{n}=0 & \text { on } & \Gamma_{N} .
\end{array}
$$

Here $\Gamma_{D}$ and $\Gamma_{N}$ are the Dirichlet and the Neumann parts of the boundary $\partial \Omega, \bar{n}$ is the outward unit normal to $\partial \Omega, K=K(x)$ is the diffusion tensor, $K=K^{T}>0, c=c(x)$ is a nonnegative function, and $f \in L_{2}(\Omega)$. We assume that $\Gamma_{D}$ is a closed subset of $\partial \Omega$ consisting of a finite number of segments (polygons) in the case $d=2(d=3)$.

For the sake of simplicity, in the paper, we consider only the case $d=2$. The extension to the three dimensional problem is basically straightforward.

The weak formulation of (1), (2) reads as follows: find $\bar{u} \in V \equiv\left\{\bar{v}: \bar{v} \in H_{\mathrm{div}}(\Omega), \int_{\partial \Omega}|\bar{v} \cdot \bar{n}|^{2} \mathrm{ds}<+\infty, \bar{v} \cdot \bar{n}=0\right.$ on $\left.\Gamma_{N}\right\}$, $p \in Q \equiv L_{2}(\Omega)$ such that

$$
\begin{aligned}
& \int_{\Omega}\left(K^{-1} \bar{u}\right) \cdot \bar{v} \mathrm{dx}-\int_{\Omega} p(\nabla \cdot \bar{v}) \mathrm{dx}=0 \\
& \int_{\Omega}(\nabla \cdot \bar{u}) q \mathrm{dx}+\int_{\Omega} c p q \mathrm{dx}=\int_{\Omega} f q \mathrm{dx}
\end{aligned}
$$

for all $(\bar{v}, q) \in V \times Q$.

Let $\Omega_{h}$ be a partitioning of $\Omega$ into $m$ nonoverlapping polygonal cells $e_{k}$ :

$$
\Omega_{h}=\bigcup_{k=1}^{m} e_{k}
$$

and $V_{h}$ and $Q_{h}$ be finite element subspaces of $V$ and $Q$, respectively. We assume that the partitioning $\Omega_{h}$ is conforming, i.e. the interface $\Gamma_{s t}$ between any adjacent cells $e_{s}$ and $e_{t}$ is always a common edge for both cells and the set $\Gamma_{N} \cap \Gamma_{D}$ belongs to the set of vertices in $\Omega_{h}$. If all the cells $e_{k}$ are triangles then $V_{h}$ can be chosen as the proper subspace of the lowest Raviart-Thomas finite element space $\operatorname{RT}_{0}\left(\Omega_{h}\right)$ (see, Brezzi and Fortin [1991]). Otherwise, we can use the new method for the construction of $V_{h}$ recently invented in Kuznetsov and Repin [2003]. The normal components $\bar{u} \cdot \bar{n}_{s t}$ of the flux $\bar{u}$ at the interfaces $\Gamma_{s t}$ between cells $e_{s}$ and $e_{t}$ are constants in both choices of $V_{h}$. Here $\bar{n}_{s t}$ denotes the unit normal to $\Gamma_{s t}$ directed from $e_{s}$ to $e_{t}$.

The mixed finite element approximation to (1), (2) reads as follows: find $\left(\bar{u}_{h}, p_{h}\right) \in V_{h} \times Q_{h}$ such that

$$
\begin{aligned}
& \int_{\Omega}\left(K^{-1} \bar{u}_{h}\right) \cdot \bar{v} \mathrm{dx}-\int_{\Omega} p_{h}(\nabla \cdot \bar{v}) \mathrm{dx}=0 \\
& \int_{\Omega}\left(\nabla \cdot \bar{u}_{h}\right) q \mathrm{dx}+\int_{\Omega} c p_{h} q \mathrm{dx}=\int_{\Omega} f q \mathrm{dx}
\end{aligned}
$$

for all $(\bar{v}, q) \in V_{h} \times Q_{h}$. 
Let $\Omega$ be splitted into two nonoverlapping subdomains $\Omega_{1}$ and $\Omega_{2}$ with a piece-wise linear simply connected interface boundary $\Gamma$. Then, the four-field macro-hybrid mixed formulation of (1), (2) originally proposed in Kuznetsov and Wheeler [1995] reads as follows: find $\left(\bar{u}_{k}, p_{k}, \lambda_{k}\right) \in V_{k} \times Q_{k} \times \Lambda_{k}, k=1,2$, $\phi \in \Phi$ such that

$$
\begin{array}{rlrl}
a_{1}\left(\bar{u}_{1}, \bar{v}_{1}\right)+b_{1}\left(p_{1}, \bar{v}_{1}\right)+c_{1}\left(\lambda_{1}, \bar{v}_{1}\right) & =0 \\
a_{2}\left(\bar{u}_{2}, \bar{v}_{2}\right)+b_{2}\left(p_{2}, \bar{v}_{2}\right)+c_{2}\left(\lambda_{2}, \bar{v}_{2}\right) & =0 \\
& & =l_{1}\left(q_{1}\right) \\
b_{1}\left(q_{1}, \bar{u}_{1}\right)-\sigma_{1}\left(p_{1}, q_{1}\right) & =l_{2}\left(q_{2}\right) \\
b_{2}\left(q_{2}, \bar{u}_{2}\right)-\sigma_{2}\left(p_{2}, q_{2}\right) & \\
c_{1}\left(\mu_{1}, \bar{u}_{1}\right) & +d_{1}\left(\phi, \mu_{1}\right) & =0 \\
c_{2}\left(\mu_{2}, \bar{u}_{2}\right) & +d_{2}\left(\phi, \mu_{1}\right) & =0 \\
d_{1}\left(\psi, \lambda_{1}\right)+d_{2}\left(\psi, \lambda_{2}\right) & =0
\end{array}
$$

for all $\left(\bar{v}_{k}, p_{k}, \mu_{k}\right) \in V_{k} \times Q_{k} \times \Lambda_{k}, k=1,2, \psi \in \Psi$.

Here

$$
\begin{aligned}
& V_{k}=\left\{\bar{v}: \bar{v} \in H_{\mathrm{div}}\left(\Omega_{k}\right), \int_{\partial \Omega_{k}}\left(\bar{v} \cdot \bar{n}_{k}\right)^{2} \mathrm{ds}<+\infty, \bar{v} \cdot \bar{n}=0 \text { on } \partial \Omega_{k} \cap \Gamma_{N}\right\}, \\
& Q_{k}=L_{2}\left(\Omega_{k}\right), \quad \Lambda_{k}=L_{2}(\Gamma), \quad k=1,2 \\
& \Phi=L_{2}(\Gamma)
\end{aligned}
$$

and

$$
\begin{aligned}
& a_{k}(\bar{u}, \bar{v})=\int_{\Omega_{k}}\left(K^{-1} \bar{u}\right) \cdot \bar{v} \mathrm{dx}, \quad \sigma_{k}(p, q)=\int_{\Omega_{k}} c p q \mathrm{dx} \\
& b_{k}(p, \bar{v})=-\int_{\Omega_{k}} p(\nabla \cdot \bar{v}) \mathrm{dx}, \quad c_{k}(\lambda, \bar{v})=(-1)^{k-1} \int_{\Gamma} \lambda\left(\bar{v} \cdot \bar{n}_{\Gamma}\right) \mathrm{ds}, \\
& d_{k}(\phi, \mu)=(-1)^{k} \int_{\Gamma} \phi \mu \mathrm{ds}, \quad l_{k}(q)=-\int_{\Omega_{k}} f q \mathrm{dx},
\end{aligned}
$$

$k=1,2$, where $\bar{n}_{\Gamma}$ is the unit normal vector to $\Gamma$ directed from $\Omega_{1}$ to $\Omega_{2}$.

Let $\Omega_{k, h}$ be a partitioning of $\Omega_{k}$ into $m_{k}$ polygons $e_{i}^{(k)}, k=1,2$. We assume that both partitionings are conformal and the set of vertices of $\Gamma$ belongs to the set of vertices of both partitionings $\Omega_{1, h}$ and $\Omega_{2, h}$. Subspaces $V_{k, h}$ and $Q_{k, h}$ of the spaces $V_{k}$ and $Q_{k}$, respectively, are defined similar to $V_{h}$ and $Q_{h}$ in problem (5). 
Let $\Gamma_{h}^{(k)}=\bigcup_{i=1}^{n_{k}} \gamma_{i, h}^{(k)}$ be the trace of $\Omega_{k, h}$ onto $\Gamma$ where $\gamma_{i, h}^{(k)}$ are the edges of the cells in $\Omega_{k, h}$ adjacent to $\Gamma, i=\overline{1, n_{k}}, k=1,2$. Here $n_{k}$ is the number of cells' edges in $\Omega_{h}$ belonging to $\Gamma, k=1,2$. We define $\Lambda_{k, h}$ by

$$
\Lambda_{k, h}=\left\{\lambda: \lambda=\text { const on } \gamma_{i, h}^{(k)}, i=\overline{1, n_{k}}\right\}
$$

$k=1,2$, and choose

$$
\Phi_{h}=\Lambda_{1, h} .
$$

The finite element approximation to (6)-(8) reads as follows: find $\left(\bar{u}_{k, h}, p_{k, h}, \lambda_{k, h}\right) \in V_{k, h} \times Q_{k, h} \times \Lambda_{k, h}, k=1,2, \phi_{h} \in \Phi_{h}$, such that the equations (6) with $\bar{u}_{k}=\bar{u}_{k, h}, p_{k}=p_{k, h}, \lambda_{k}=\lambda_{k, h}, k=1,2, \phi=\phi_{h}$ are satisfied for all $\left(\bar{v}_{k}, q_{k}, \mu_{k}\right) \in V_{k, h} \times Q_{k, h} \times \Lambda_{k, h}, k=1,2, \psi \in \Phi_{h}$. This approximation results in the system

$$
\mathcal{A}\left(\begin{array}{c}
w_{1} \\
w_{2} \\
\phi
\end{array}\right)=\left(\begin{array}{c}
F_{1} \\
F_{2} \\
0
\end{array}\right)
$$

with the matrix

$$
\mathcal{A}=\left(\begin{array}{ccc}
A_{1} & 0 & D_{1}^{T} \\
0 & A_{2} & D_{2}^{T} \\
D_{1} & D_{2} & 0
\end{array}\right)
$$

where

$$
A_{k}=\left(\begin{array}{ccc}
M_{k} & B_{k}^{T} & C_{k}^{T} \\
B_{k} & -\Sigma_{k} & 0 \\
C_{k} & 0 & 0
\end{array}\right)
$$

are the saddle point matrices, $k=1,2$, and

$$
w_{k}=\left(\begin{array}{l}
u_{k} \\
p_{k} \\
\lambda_{k}
\end{array}\right), \quad F_{k}=\left(\begin{array}{c}
0 \\
-f_{k} \\
0
\end{array}\right), \quad k=1,2 .
$$

Here $M_{k}$ is a symmetric positive definite matrix, and $\Sigma_{k}$ is a symmetric positive definite (or semidefinite) matrix, $k=1,2$.

\section{Hybridization and condensation}

The extension of (6)-(8) to the case of many subdomains is straightforward. We consider the hybrid mixed formulation based on partitionings of $\Omega_{k, h}$ into subdomains/cells $e_{i}^{(k)}$ used in Sect. 2 for the approximation of the problem (6)-(8). 
We introduce new spaces $V_{k, i, h}$ and $Q_{k, i, h}$ to be the restrictions onto $e_{i}^{(k)}$ of $V_{k, h}$ subject to $\Gamma_{N}=\emptyset$ and $Q_{k, h}$, respectively, $i=\overline{1, m_{k}}, k=1,2$, and define the spaces $\widehat{V}_{k, h}$ and $\widehat{Q}_{k, h}$ as the products of the spaces $V_{k, i, h}$ and $Q_{k, i, h}$, $i=\overline{1, m_{k}}$, respectively, $k=1,2$. Then, we introduce spaces $\Lambda_{k, i, h}$ of functions $\lambda$ defined on $\partial e_{i}^{(k)}$ which are constants on each interface $\Gamma_{k, i, h}$ between $e_{i}^{(k)}$ and adjacent cells $e_{j}^{(k)}$ as well as on the intersections $\partial e_{i}^{(k)}$ with the linear parts of $\partial \Omega, i=\frac{j}{1, m_{k}}, k=1,2$. The functions in $\Lambda_{k, i, h}$ should vanish on $\Gamma_{D}$. We denote by $\widehat{\Lambda}_{k, h}$ the product of all spaces $\Lambda_{k, i, h}, k=1,2$. Finally, we preserve the definition for $\Phi_{h}$ from Sect. 2 .

The new finite element problem reads as follows: find $\left(\hat{u}_{k, h}, \hat{p}_{k, h}, \hat{\lambda}_{k, h}\right) \in$ $\widehat{V}_{k, h} \times \widehat{Q}_{k, h} \times \widehat{\Lambda}_{k, h}, k=1,2, \hat{\phi}_{h} \in \Phi_{h}$, such that

$$
\begin{aligned}
& \hat{a}_{1}\left(\hat{u}_{1, h}, \bar{v}_{1}\right)+\hat{b}_{1}\left(\hat{p}_{1, h}, \bar{v}_{1}\right)+\hat{c}_{1}\left(\hat{\lambda}_{1, h}, \bar{v}_{1}\right)=0 \\
& \hat{a}_{2}\left(\hat{u}_{2, h}, \bar{v}_{2}\right)+\hat{b}_{2}\left(\hat{p}_{2, h}, \bar{v}_{2}\right)+\hat{c}_{2}\left(\hat{\lambda}_{2, h}, \bar{v}_{2}\right)=0 \\
& \hat{b}_{1}\left(q_{1}, \hat{u}_{1, h}\right)-\hat{\sigma}_{1}\left(\hat{p}_{1, h}, q_{1}\right) \quad=\hat{l}_{1}\left(q_{1}\right) \\
& \hat{b}_{2}\left(q_{2}, \hat{u}_{2, h}\right)-\hat{\sigma}_{2}\left(\hat{p}_{2, h}, q_{2}\right) \quad=\hat{l}_{2}\left(q_{2}\right) \\
& \hat{c}_{1}\left(\mu_{1}, \hat{u}_{1, h}\right) \quad+\hat{d}_{1}\left(\hat{\phi}_{h}, \mu_{1}\right)=0 \\
& \hat{c}_{2}\left(\mu_{2}, \hat{u}_{2, h}\right)+\hat{d}_{2}\left(\hat{\phi}_{h}, \mu_{1}\right)=0 \\
& \hat{d}_{1}\left(\psi, \hat{\lambda}_{1, h}\right)+\hat{d}_{2}\left(\psi, \hat{\lambda}_{2, h}\right)=0
\end{aligned}
$$

for all $\left(\bar{v}_{k}, p_{k}, \mu_{k}\right) \in \widehat{V}_{k, h} \times \widehat{Q}_{k, h} \times \widehat{\Lambda}_{k, h}, k=1,2, \psi \in \Phi_{h}$.

Here,

$$
\begin{aligned}
& \hat{a}_{k}\left(\bar{u}_{k}, \bar{v}_{k}\right)=\sum_{i=1}^{m_{k}} \int_{e_{i}^{(k)}}\left(K^{-1} \bar{u}_{k, i}\right) \cdot \bar{v}_{k, i} \mathrm{dx}, \quad \hat{b}_{k}\left(p_{k}, \bar{v}_{k}\right)=-\sum_{i=1}^{m_{k}} \int_{e_{i}^{(k)}} p_{k, i}\left(\nabla \cdot \bar{v}_{k, i}\right) \mathrm{dx}, \\
& \hat{c}_{k}\left(\lambda_{k}, \bar{v}_{k}\right)=\sum_{i=1}^{m_{k}} \int_{\partial e_{i}^{(k)} \backslash \Gamma_{D}} \lambda_{k, i}\left(\bar{v}_{k, i} \cdot \bar{n}_{k, i}\right) \mathrm{ds}, \quad \hat{\sigma}_{k}\left(p_{k}, q_{k}\right)=\sum_{i=1}^{m_{k}} \int_{e_{i}^{(k)}} c p_{k, i} q_{k, i} \mathrm{dx}, \\
& \hat{d}_{k}\left(\phi, \mu_{k}\right)=(-1)^{k} \sum_{i=1}^{m_{k}} \int_{\Gamma \cap \partial e_{i}^{(k)}} \phi \mu_{k, i} \mathrm{ds}, \quad \hat{l}_{k}\left(q_{k}\right)=-\sum_{i=1}^{m_{k}} \int_{e_{i}^{(k)}} f q_{k, i} \mathrm{dx},
\end{aligned}
$$

where $\bar{n}_{k, i}$ is the outward unit normal to $\partial e_{i}^{(k)}, i=\overline{1, m_{k}}, k=1,2$.

The finite element problem (15), (16) is said to be the hybridization of the finite element problem of the previous Section. It can be proved that the problems are equivalent, i.e. the restrictions of $\bar{u}_{k, h}$ and $p_{k, h}$ onto a cell $e_{i}^{(k)}$ coincide with $\hat{u}_{k, i, h}$ and $\hat{p}_{k, i, h}$, respectively, $\lambda_{k, h}$ coincides with restriction of $\hat{\lambda}_{k, h}$ onto $\Gamma$, and $\phi_{h}$ coincides with $\hat{\phi}_{h}$. 
Problem (15), (16) results in the system of linear algebraic equations

$$
\mathcal{A}\left(\begin{array}{c}
w_{1} \\
\lambda_{1} \\
w_{2} \\
\lambda_{2} \\
\phi
\end{array}\right)=\left(\begin{array}{c}
F_{1} \\
0 \\
F_{2} \\
0 \\
0
\end{array}\right)
$$

with the $5 \times 5$ block matrix

$$
\mathcal{A}=\left(\begin{array}{ccccc}
A_{1} & C_{1}^{T} & 0 & 0 & 0 \\
C_{1} & 0 & 0 & 0 & D_{1}^{T} \\
0 & 0 & A_{2} & C_{2}^{T} & 0 \\
0 & 0 & C_{2} & 0 & D_{2}^{T} \\
0 & D_{1} & 0 & D_{2} & 0
\end{array}\right)
$$

where $A_{k}$ is the block diagonal matrix with the diagonal blocks

$$
\begin{aligned}
& A_{k, i}=\left(\begin{array}{cc}
M_{k, i} & B_{k, i}^{T} \\
B_{k, i} & -\Sigma_{k, i}
\end{array}\right) \\
& C_{k}=\left(C_{k, 1} \ldots C_{k, m_{k}}\right) \text {, }
\end{aligned}
$$

and

$$
F_{k}=\left(\begin{array}{c}
F_{k, 1} \\
\vdots \\
F_{k, m_{k}}
\end{array}\right), \quad F_{k, i}=\left(\begin{array}{c}
0 \\
-f_{k, i}
\end{array}\right), \quad i=\overline{1, m_{k}},
$$

$k=1,2$. The subvectors $w_{1}$ and $w_{2}$ can be excluded from the system by the block Gauss elimination method. The reduced system is given by

$$
\left(\begin{array}{ccc}
S_{1} & 0 & -D_{1}^{T} \\
0 & S_{2} & -D_{2}^{T} \\
-D_{1} & -D_{2} & 0
\end{array}\right)\left(\begin{array}{c}
\lambda_{1} \\
\lambda_{2} \\
\phi
\end{array}\right)=\left(\begin{array}{c}
g_{1} \\
g_{2} \\
0
\end{array}\right)
$$

where

$$
\begin{aligned}
S_{k} & =\sum_{i=1}^{m_{k}} C_{k, i} A_{k, i}^{-1} C_{k, i}^{T} \\
g_{k} & =\sum_{i=1}^{m_{k}} C_{k, i} A_{k, i}^{-1} F_{k, i},
\end{aligned}
$$

$k=1,2$. The system (22)-(24) is said to be the condensation of the system (17)-(21). 


\section{Algebraic solvers}

The saddle point system (22) can be explicitly reduced to a system with a positive definite matrix. With additional block partitioning

$$
\begin{aligned}
& S_{k}=\left(\begin{array}{cc}
S_{k k} & S_{k \Gamma} \\
S_{\Gamma k} & S_{\Gamma \Gamma}^{(k)}
\end{array}\right), \quad D_{k}=\left(\begin{array}{ll}
0 & D_{k \Gamma}
\end{array}\right), \\
& \lambda_{k}=\left(\begin{array}{c}
\lambda_{k k} \\
\lambda_{k \Gamma}
\end{array}\right), \quad g_{k}=\left(\begin{array}{c}
g_{k 1} \\
g_{k \Gamma}
\end{array}\right)
\end{aligned}
$$

where the blocks $S_{\Gamma \Gamma}^{(k)}, D_{k \Gamma}, \lambda_{k \Gamma}$, and $g_{k \Gamma}$ correspond to the degrees of freedom located on the interface $\Gamma, k=1,2$. System (22) can be written in the form of $5 \times 5$ block system

$$
\left(\begin{array}{ccccc}
S_{11} & S_{1 \Gamma} & 0 & 0 & 0 \\
S_{\Gamma 1} & S_{\Gamma \Gamma}^{(1)} & 0 & 0 & -D_{1 \Gamma}^{T} \\
0 & 0 & S_{22} & S_{2 \Gamma} & 0 \\
0 & 0 & S_{\Gamma 2} & S_{\Gamma \Gamma}^{(2)} & -D_{2 \Gamma}^{T} \\
0 & -D_{1 \Gamma} & 0 & -D_{2 \Gamma} & 0
\end{array}\right)\left(\begin{array}{c}
\lambda_{11} \\
\lambda_{1 \Gamma} \\
\lambda_{22} \\
\lambda_{2 \Gamma} \\
\phi
\end{array}\right)=\left(\begin{array}{c}
g_{11} \\
g_{1 \Gamma} \\
g_{22} \\
g_{2 \Gamma} \\
0
\end{array}\right)
$$

In this system $D_{1 \Gamma}$ is the diagonal matrix. Then, excluding the subvectors $\lambda_{1 \Gamma}$ and $\phi$ by the block Gauss elimination method we get the system

$$
R\left(\begin{array}{c}
\lambda_{11} \\
\lambda_{22} \\
\lambda_{2 \Gamma}
\end{array}\right)=\left(\begin{array}{l}
g_{11} \\
g_{22} \\
g_{\Gamma}
\end{array}\right)
$$

with the symmetric positive definite matrix

$$
R=\left(\begin{array}{ccc}
S_{11} & 0 & R_{1 \Gamma}^{T} \\
0 & S_{22} & S_{2 \Gamma} \\
R_{1 \Gamma} & S_{\Gamma 2} & R_{\Gamma \Gamma}
\end{array}\right)
$$

where

$$
\begin{aligned}
& R_{\Gamma \Gamma}=S_{\Gamma \Gamma}^{(2)}+D_{2 \Gamma}^{T} D_{1 \Gamma}^{-1} S_{\Gamma \Gamma}^{(1)} D_{1 \Gamma}^{-1} D_{2 \Gamma}, \\
& R_{1 \Gamma}=-D_{2 \Gamma}^{T} D_{1 \Gamma}^{-1} S_{\Gamma 1}, \\
& g_{\Gamma}=g_{2 \Gamma}-D_{2 \Gamma}^{T} D_{1 \Gamma}^{-1} g_{1 \Gamma} .
\end{aligned}
$$

To solve the system (27) we can use iterative techniques developed for algebraic systems with symmetric positive definite matrices. We recall that for the mortar $P_{1}$ finite element methods the above explicit reduction is not applicable.

The preconditioned Lanczos method is a good candidate to solve the saddle point system (22). In Kuznetsov [1995] an efficient preconditioner was 
proposed for the $P_{1}$ mortar element method. By coupling the ideas from Kuznetsov [1995], Kuznetsov and Wheeler [1995] with the new results from the recent publication by Kuznetsov [2003] we are able to derive efficient preconditioners for the system matrix in (22) as well. This is a topic for another publication.

Acknowledgement. The author is thankful to Oleg Boyarkin for his help in preparation of the paper.

\section{References}

F. Brezzi and M. Fortin. Mixed and Hybrid Finite Element Methods. SpringerVerlag, New York - Berlin - Heidelberg, 1991.

Y. A. Kuznetsov. Efficient iterative solvers for elliptic problems on nonmatching grids. Russ. J. Numer. Anal. Math. Modelling, 10(3):187-211, 1995.

Y. A. Kuznetsov. Spectrally equivalent preconditioners for mixed hybrid discretizations of diffusion equations on distorted meshes. J. Numer. Math., 11(1):61-74, 2003.

Y. A. Kuznetsov and S. K. Repin. New mixed finite element method on polygonal and polyhedral meshes. Russ. J. Numer. Anal. Math. Modelling, 18(3):261-278, 2003.

Y. A. Kuznetsov and M. F. Wheeler. Optimal order substructuring preconditioners for mixed finite element methods on nonmatching grids. East-West J. Numer. Math., 3(2):127-143, 1995. 